\title{
Origin of Mammalian Biliprotein and Rearrangement of Bilirubin Glucuronides In Vivo in the Rat
}

\author{
Antony F. McDonagh and Lucita A. Palma \\ San Francisco, California 94143 \\ John J. Lauff and Tai-Wing Wu \\ Research Laboratories, Eastman Kodak Company, \\ Rochester, New York 14640
}

Gastroenterology Unit and Liver Center, University of California,

bstract. In hepatobiliary disease bilirubin becomes bound covalently to serum albumin, producing a nondissociable bile pigment-protein complex (biliprotein). To elucidate the mechanism of biliprotein formation we studied the bile pigment composition of blood from animals with experimental cholestasis and carried out comparative studies on the rate of biliprotein formation in vivo and in vitro during incubation of bilirubin glucuronides with albumin. Bile duct ligation in the rat and guinea pig led to rapid accumulation in the circulation of bilirubin, heterogeneous bilirubin esters of glucuronic acid, and a biliprotein that migrated along with albumin on high performance liquid chromatography. When the obstruction was removed, biliprotein remained longer in the circulation than did the other bile pigment species. Biliprotein and heterogeneous bilirubin esters of glucuronic acid were not formed in bile duct-ligated homozygous Gunn rats but they were formed when bilirubin glucuronides were incubated with Sprague-Dawley rat serum or human serum albumin at $37^{\circ} \mathrm{C}$ in vitro. Bilirubin glucuronide rearrangement in vitro was accompanied by nonenzymic hydrolysis. We conclude that the formation of biliprotein in vivo is probably nonenzymic and suggest that mammalian biliprotein is formed by acyl migration of bilirubin from a bilirubin-glucuronic acid ester to a nucleophilic site on albumin.

\section{Introduction}

Under certain pathological conditions abnormal amounts of bilirubin $^{1}$ and its metabolites occur in the circulation, either

Received for publication 28 December 1983 and in revised form 14 May 1984.

J. Clin. Invest.

(c) The American Society for Clinical Investigation, Inc. 0021-9738/84/09/0763/08

Volume 74, September 1984, 763-770 free or reversibly associated with albumin. Measurement of these in body fluids is useful clinically, particularly in the detection and diagnosis of hepatobiliary disease (1). It is widely believed that four main bilirubinoid molecular species occur in humans: bilirubin, bilirubin $\beta-1-O$-acyl diglucuronide $(\mathrm{BDG})^{2}$, and two isomeric bilirubin $\beta$-1- $O$-acyl monoglucuronides (BMG). However, this view is incorrect and oversimplified for two reasons. First, 1-O-acyl glucuronides are unstable and isomerize to 2-, 3- and 4-O-acyl esters of glucuronic acid at physiological pH (2-7). Consequently, icteric blood plasma may contain not only $\beta-1-O$-acyl glucuronides of bilirubin, but isomers of these (3-5). Second, there is longstanding evidence that proteins containing irreversibly bound bile pigment occur in some jaundiced sera (8-13). Such biliproteins are not only of interest apropos of liver disease, but also with respect to the structure and biosynthesis of nonmammalian biliproteins, which are widely distributed in nature. Currently little is known about the kinetics of bilirubin glucuronide isomerization in vivo or the metabolic origin of mammalian biliproteins. We now present evidence that these processes are temporally related in vivo and in vitro and that the formation of biliproteins in mammals is probably nonenzymic.

\section{Methods}

Animal studies. Sprague-Dawley (SD) rats and guinea pigs were from Simonsen Laboratories, Gilroy, CA. Homozygous Gunn rats were bred at the University of California, San Francisco. For bile duct ligations, the common duct was ligated in two places and cut between the ties. Except where noted, blood samples were obtained from the heart or abdominal aorta. Total bilirubin concentrations were estimated by a

1. Throughout this paper, bilirubin refers to the IX $\alpha$ isomer only. The terms conjugated bilirubin and bilirubin glucuronide refer only to enzymatically formed 1-O-acyl glucuronic acid esters of bilirubin. They do not include rearranged isomers of bilirubin $\beta-1-O$-acyl diglucuronide or bilirubin $\beta-1-O$-acyl monoglucuronides or substances containing bilirubin or its derivatives bonded covalently to protein. 2. Abbreviations used in this paper: BDG, bilirubin $\beta-1-O$-acyl diglucuronide; BMG, bilirubin $\beta$-1-O-acyl monoglucuronides; HPLC, highperformance liquid chromatography; HSA, human serum albumin; SD, Sprague-Dawley. 
diazo method (14) and by high-performance liquid chromatography (HPLC) (12).

In vitro studies. Human serum albumin (HSA) was Fraction $\mathrm{V}$ from Miles Laboratories, Inc., (Elkhart, IN). Crude 1-O-acyl glucuronides (BMG/BDG) were isolated from fresh rat bile by solvent extraction (15) with precautions to avoid acyl migration and disproportionation. Reversed-phase HPLC of this preparation with $0.1 \mathrm{M}$ methanolic di$n$-dodecylamine acetate (16) showed it to contain $>82 \%$ BMG/BDG, with $\sim 7 \%$ of rearranged glucuronides and no significant III $\alpha$ and XIII $\alpha$ glucuronides. Purified BDG was a gift from Dr. E. Gordon (McGill University, Montreal). Reversed-phase HPLC of this material showed it to contain $12 \%$ bilirubin XIII $\alpha$ diglucuronide, 67\% BDG, and $16 \%$ bilirubin III $\alpha$ diglucuronide with $<3 \%$ BMG. Solutions of bilirubin conjugates were prepared at $0^{\circ} \mathrm{C}$ under safelights and, after zero-time samples were removed, incubated at $37^{\circ} \mathrm{C}$ in closed tubes in the dark. Solutions in aqueous HSA (0.5 mM) were degassed and incubated under argon. The pH of unbuffered HSA solutions was adjusted with $1 \mathrm{M} \mathrm{NaOH}$ or $\mathrm{HCl}$.

HPLC. Two complementary reversed-phase systems were used (12, 16). In system $A$ the mobile phase was $0.1 \mathrm{M}$ methanolic di- $n$ octylamine acetate (16). Samples, diluted 1:9 or 1:4 with mobile phase at $0^{\circ} \mathrm{C}$, were centrifuged to remove protein and injected via a $20 \mu \mathrm{l}$ loop. System B has been described by Lauff et al. (12). For practical reasons samples $(0.5-1.0 \mathrm{ml})$ to be analyzed on system $B$ were frozen immediately after collection and lyophilized. After transport and brief storage at $<-5^{\circ} \mathrm{C}$ in the dark the samples were reconstituted with water, treated with $\mathrm{HSA}$ and $\mathrm{Na}_{2} \mathrm{SO}_{4}$ to remove globulins (12), and analyzed double-blind in duplicate. System A separates bilirubin, BDG, and BMG but does not resolve the two BMG isomers (Fig. $1 a$ ). Only pigments readily dissociable from albumin are detected and biliproteins are lost with the protein precipitate. In contrast, system B resolves bilirubin, bilirubin monoconjugates, bilirubin diconjugates, and biliprotein (or pigment co-eluting with albumin) into four main single peaks (Fig. 2, $b$ and $c$ ) (12).

\section{Results}

In vivo studies. Cholestasis was induced in four adult male SD rats by ligation of the bile duct, and tail-vein serum samples were analyzed periodically by HPLC system A (Fig. $1, b-f$ ). Also analyzed were single serum samples from 14 other SD rats whose bile ducts had been obstructed for 16 to $96 \mathrm{~h}$ as follows: $16 \mathrm{~h}(n=2), 48 \mathrm{~h}(n=9), 72 \mathrm{~h}(n=2)$, and $96 \mathrm{~h}(n$ $=1$ ). Serum collected before ligation was essentially devoid of bile pigment, but by $2 \mathrm{~h} \mathrm{BDG}$ and BMG were clearly present in addition to unconjugated bilirubin. After $6 \mathrm{~h}$ the two glucuronide peaks had increased substantially and a less mobile shoulder was perceptible on the edge of the BMG peak. By 24 $\mathrm{h}$ this shoulder had become a distinct peak centered at $\sim 7.6$ min, and by $72 \mathrm{~h}$ it surpassed the BMG peak. Although this peak was moderately sharp on HPLC system A, it broadened and became partly resolved into several overlapping peaks when the reversed-phase chromatography was carried out by the use of solvents of higher resolving power such as $0.1 \mathrm{M}$ methanolic di- $n$-dodecylamine acetate (16) or $0.1 \mathrm{M}$ methanolic di- $n$-octylamine acetate containing $8 \%$ water (data not shown). Therefore, the peak at $\sim 7.6$ min which follows the BMG peak represents an unresolved mixture of pigments. The BDG peak
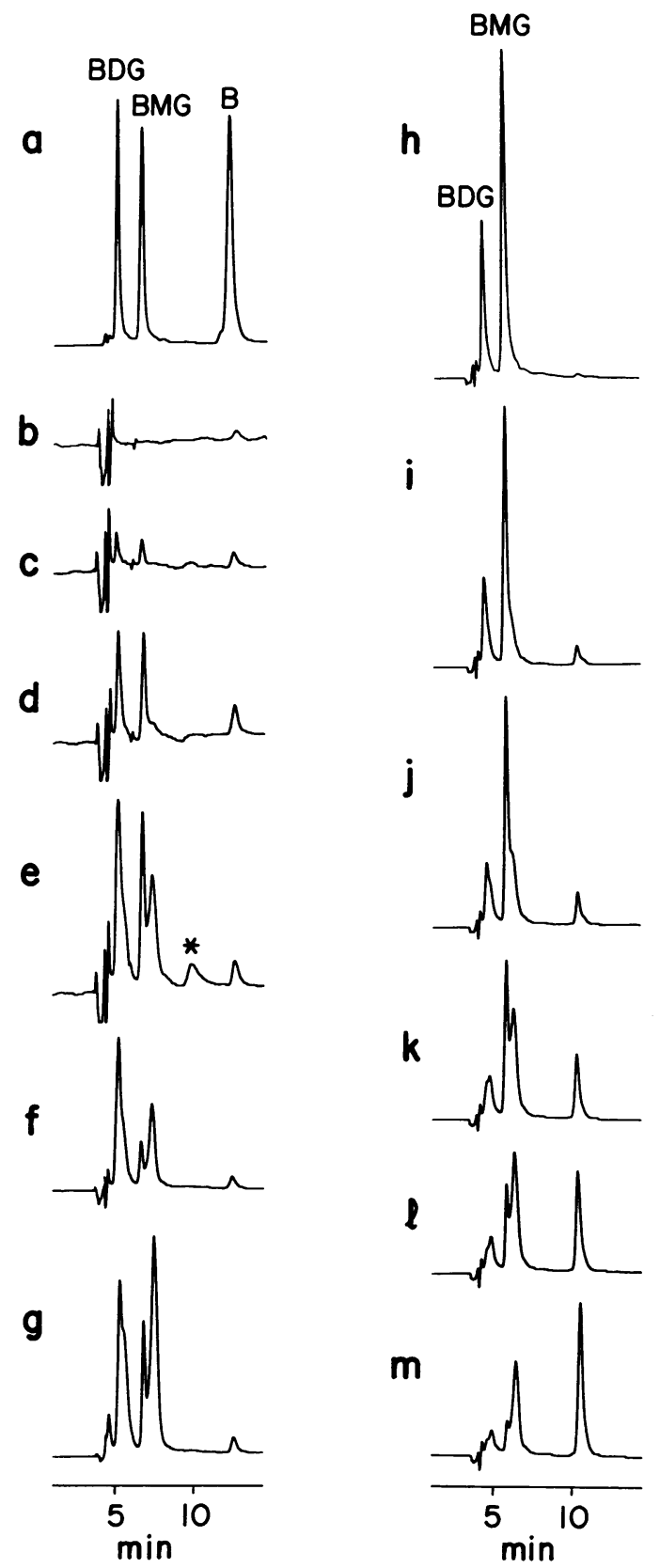

Figure 1. HPLC of natural and synthetic bilirubin glucuronic acid esters with system A. (a) Authentic BMG, BDG, and bilirubin (B). $(b-f)$ Serum from SD rat after $0,2,6,24$, and $72 \mathrm{~h}$, respectively, of bile duct ligation (note: the vertical scale is reduced in $f$ ). (g) Serum from human patient. ( $h$ ) Crude BMG/BDG from rat bile $(16 \mathrm{mg} / 100$ $\mathrm{ml}$ ) in $0.5 \mathrm{mM}$ HSA, pH 6.8. $(i-m)$ Solution $h$ after incubation for $6 \mathrm{~h}, 37^{\circ} \mathrm{C}$, at $\mathrm{pH} 6.8-7.0,7.2-7.5,7.7-8.0,8.0-8.5$, and 8.4-9.0, respectively (initial and final $\mathrm{pH}$ values given). ${ }^{*}$ Heme.

also showed marked broadening in samples collected after 24 h or more of cholestasis (Fig. 1, $e$ and $f$ ). Proof that this broadening was due to unresolved overlapping peaks was 
obtained by chromatography with the solvents of higher resolving power noted above. Therefore, cholestasis leads to accumulation in the circulation of not only BMG and BDG, but also of two groups of protein-dissociable pigments that are different from and predominantly less polar than BMG and BDG, respectively. Based on previous studies (3-5), our in vitro studies described below, and arguments summarized in the Discussion, we assign these multiple peaks eluting close to BMG and BDG in system A to constitutional isomers of BMG and BDG formed by reversible intramolecular migration of bilirubin from the C-1 to the C-2, C-3, and C-4 hydroxyls of the glucuronic aid substituent. We will refer to these mutant bilirubin esters collectively as altered glucuronides. ${ }^{3}$

Except for variations in the proportion of altered glucuronides to $1-O$-acyl glucuronides, qualitatively similar chromatograms were obtained with serum from $16 \mathrm{SD}$ rats after $16 \mathrm{~h}$ to 2 wk of cholestasis. Serum from a human patient with hemolysis and autoimmune liver disease also showed an HPLC pattern that was strikingly similar to that observed in rats (Fig. $1 \mathrm{~g}$ ). As expected, BDG, BMG, and altered glucuronides were undetectable in serum from homozygous Gunn rats deficient in bilirubin glucuronyl transferase even up to $72 \mathrm{~h}$ after ligation of the bile duct $(n=6)$.

Reconstituted serum samples $(n=11)$ from nine SD rats with bile ducts ligated for $>24 \mathrm{~h}$ were analyzed by chromatography with HPLC system B. Heterogeneity of the main bilirubin glucuronic acid ester bands was not observed, although there was some slight peak broadening of the bilirubin monoglucuronic acid peak in particular. Four main peaks were detected, as previously observed with cholestatic human serum $(12,13)$, corresponding to biliprotein, bilirubin diglucuronic esters, bilirubin monoglucuronic esters, and bilirubin, respectively (Fig. 2). Biliprotein was detected in all SD samples collected within 1-14 d of cholestasis (Table I) in concentrations of 0.7-4.6 $\mathrm{mg} / 100 \mathrm{ml}$. The proportion of pigment present as biliprotein increased with duration of cholestasis in sequentially drawn

3. For each of the two positional isomers of BMG, intramolecular migration of the bilirubin acyl moiety can lead to three constitutional isomers, each of which can exist in at least two isomeric forms owing to anomerization of the $-\mathrm{OH}$ group at $\mathrm{C}-1$ of the sugar. In addition, reverse migration of the bilirubin acyl group from the C-2 -OH to an anomerized $(\alpha)-\mathrm{OH}$ group at $\mathrm{C}-1$ can lead to another pair of constitutional isomers. There are, therefore, a total of 16 possible isomers of bilirubin IX $\alpha \boldsymbol{O}$-acyl monoglucuronic acid ester. Similarly, intramolecular rearrangement of BDG can lead to an additional 63 isomers of the IX $\alpha$ type. Consequently, cholestatic serum might well contain up to 80 chemically distinct glucuronic acid esters of bilirubin IX $\alpha$. None of the 77 possible rearrangement products of BMG and BDG has been purified or characterized. Their existence in mixtures has been deduced from extensive structural studies on dipyrrolic azo derivatives and by lack of reactivity to $\beta$-glucuronidase (3-5). By convention, only the 1-O acyl esters of glucuronic acid, and not the 2-, 3-, and 4-O-acyl esters, are called glucuronides. Intramolecular rearrangement of BMG and BDG could also lead to other types of isomers (e.g., orthoesters) that are not acyl esters. However, these have never been detected.

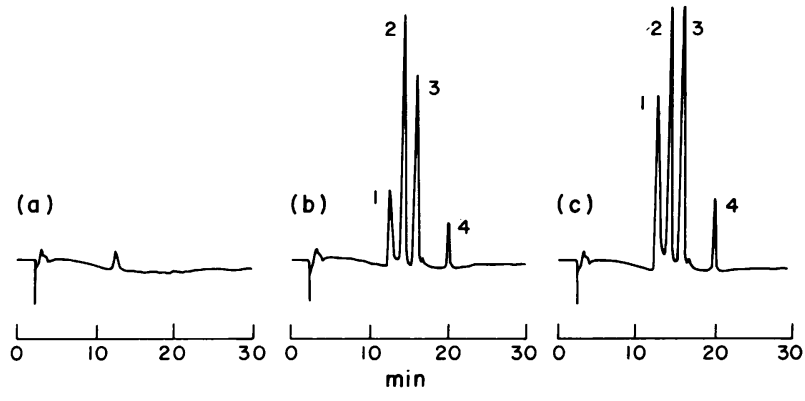

Figure 2. HPLC of serum from SD rats before and after bile duct ligation by the use of system B with detection at $436 \mathrm{~nm}$. (a) Before ligation; (b) after a 48-h ligation; (c) after a 96-h ligation. Peak 1, biliprotein; peak 2, diglucuronic acid esters of bilirubin; peak 3, monoglucuronic acid esters of bilirubin; peak 4 , bilirubin. The small peak near $12.1 \mathrm{~min}$ in $a$ is a background peak, due to weak absorbance by albumin at $436 \mathrm{~nm}$ (11). (Traced from original chromatograms.)

samples from the same animal and tended to increase with duration of cholestasis for the group as a whole (Table I). Biliprotein was undetectable in serum from healthy or shamoperated SD rats $(n=5)$ and undetectable or $<1 \%$ of total pigment in serum from Gunn rats before $(n=2)$ and after 48 $(n=1)$ or $72 \mathrm{~h}(n=2)$ of cholestasis. Three of four guinea pigs died within $72 \mathrm{~h}$ of bile duct ligation. In the animal that

Table I. Biliprotein and Dissociable Bile

Pigments in Rat Serum After Bile Duct Ligation

\begin{tabular}{|c|c|c|c|c|c|c|}
\hline \multirow[b]{2}{*}{ Time } & \multicolumn{2}{|c|}{ Total bilirubin } & \multicolumn{4}{|c|}{$\%$ Composition ${ }^{*} \ddagger$} \\
\hline & HPLC* & Diazo & $\delta(\overline{\mathrm{M}}$ & $\overline{\text { ean) }}$ & $\beta+\gamma$ & $\alpha$ \\
\hline$d$ & $\mathrm{mg} / 100 \mathrm{ml}$ & $\mathrm{mg} / 100 \mathrm{ml}$ & & & & \\
\hline 1 & - & 4.6 & 16 & & 75 & 10 \\
\hline $2 \S$ & 7.0 & 6.0 & 18 & & 76 & 6 \\
\hline $2^{\|}$ & 12 & 10.0 & 13 & $(\overline{17})$ & 80 & 6 \\
\hline 2 & - & 9.0 & 20 & & 71 & 9 \\
\hline 3 & - & 11.0 & 26 & & 47 & 27 \\
\hline 3 & $9.9 \pi$ & - & 19 & $(\overline{22})$ & 65 & 19 \\
\hline 3 & 8.8 & - & 20 & & 66 & 14 \\
\hline $4^{\prime \prime}$ & 22 & 22 & 21 & $(\overline{23})$ & 74 & 6 \\
\hline $4 \S$ & 7.8 & 9.9 & 25 & & 71 & 3 \\
\hline 14 & - & 10.0 & 37 & $(\overline{33})$ & 58 & 4 \\
\hline 14 & - & 9.9 & 28 & & 68 & 4 \\
\hline
\end{tabular}

* By HPLC, system B.

$\ddagger \delta$, biliprotein; $\beta$ and $\gamma$, bilirubin mono- and diglucuronic acid esters; $\alpha$, bilirubin.

\$ Sequential samples from the same animal.

"Sequential samples from the same animal.

II Dilution of this sample with $0.1 \mathrm{M}$ methanolic di- $n$-octylamine acetate and analysis of the washed and reconstituted precipitate on HPLC system B gave $100 \%$ biliprotein with a trace of conjugates. 
survived, $18 \%$ of the total serum bile pigment $(10 \mathrm{mg} / 100 \mathrm{ml})$ was present as biliprotein after $48 \mathrm{~h}$ of cholestasis. The human serum sample that gave the chromatogram shown in Fig. $1 \mathrm{~g}$ contained $45 \mathrm{mg} / 100 \mathrm{ml}$ total bilirubin (by diazo), of which $30 \%$ was found to be biliprotein by HPLC in system B.

Clearance of endogenous biliprotein and protein-dissociable pigments was studied in nine SD rats fitted with indwelling bile duct cannulas (17). Initially, the free tip of the cannula was sealed and sutured within the abdomen. After $48 \mathrm{~h}$ of cholestasis a tail-vein blood sample from each animal was analyzed by HPLC with system A. All showed the presence of substantial quantities of bilirubin glucuronides and altered glucuronides. The cannula was then brought through the skin, the tip was cut off, and bile was allowed to drain freely to the outside, with the animal in a restraining cage. During the drainage period, tail-vein blood samples from three of the rats were analyzed periodically by HPLC with system A. These analyses revealed a rapid loss of bilirubin glucuronides and altered glucuronides from the circulation during the first $2 \mathrm{~h}$ of bile drainage, with essentially complete disappearance within $7 \mathrm{~h}$, which is consistent with previous studies (18). After 7-12 $h$ of biliary drainage, all nine rats were exsanguinated and serum was analyzed by the diazo reaction for total bilirubin (i.e., biliprotein + bilirubin + bilirubin glucuronides + altered glucuronides) and by HPLC with systems A and B. Clearance of protein-dissociable pigments for each animal was checked with system A by a comparison of the chromatogram of serum obtained by exsanguination with the chromatogram of serum obtained from the tail vein just before relief of cholestasis. Six of the animals showed very low levels of protein-dissociable pigments. The remaining three showed somewhat higher amounts, probably because of some restriction of bile flow toward the end of the drainage period or during the exsanguination procedure. HPLC of the nine final serum samples with system B showed that all contained a substantial proportion of biliprotein (Table II). The mean percentage of pigment present as biliprotein for the whole group was $60 \%$, much higher than the $22 \%$ mean value observed in rats with unrelieved obstruction (Table I). Furthermore, when the samples were ranked, as in Table II, in order of decreasing dissociable pigment concentration based on HPLC with system A, the proportion of pigment present as biliprotein was found to increase as the total bilirubin and the amount of dissociable pigment decreased. In two samples, biliprotein was the only pigment detectable. Biliprotein was not detectable in shamoperated controls $(n=2)$. These data show that biliprotein becomes the major bilirubin pigment present in the circulation as hyperbilirubinemia due to cholestasis diminishes. They also suggest that biliprotein is cleared less rapidly from the circulation than are bilirubin and its glucuronic acid esters, as would be expected.

In vitro studies. Methods for preparing or isolating BMG, BDG, or their isomers as analytically pure, isomerically homogeneous solids are not available. For in vitro studies we used a crude BMG/BDG preparation extracted from rat bile, purified BDG from rat bile, and conjugated bilirubin isolated
Table II. Biliprotein and Dissociable Bile

Pigments in Postcholestatic Rat Serum

\begin{tabular}{lllll}
\hline & & \multicolumn{3}{c}{ \% Composition } \\
\cline { 4 - 5 } Rat number & Total bilirubin $¥$ & $\delta$ & $\beta+\gamma$ & $\alpha$ \\
\hline & $m g / 100 \mathrm{ml}$ & & & \\
1 & 7.2 & 24 & 66 & 10 \\
2 & 4.4 & 32 & 57 & 11 \\
3 & 2.4 & 20 & 65 & 15 \\
4 & 1.9 & 55 & 27 & 12 \\
5 & 1.7 & 72 & 10 & 18 \\
6 & 1.7 & 70 & 16 & 14 \\
7 & 0.9 & 67 & 0 & $33 \S$ \\
8 & 0.9 & 100 & 0 & 0 \\
9 & 1.0 & 100 & 0 & 0
\end{tabular}

The bile duct of each animal was obstructed for $48 \mathrm{~h}$. The obstruction was then removed and 7-12 h later the rats were exsanguinated and serum was analyzed by HPLC. In the Table the rats are ranked in order of decreasing dissociable bile pigment level based on HPLC with system $A$.

* By HPLC, system B (12). $\delta$, biliprotein; $\beta+\gamma$, total bilirubin glucuronic acid esters; $\alpha$, bilirubin.

₹ By diazo.

$\S$ Close to limits of accurate quantitative estimation.

from human bile by the procedure of Wu et al. (19). The first two of these preparations appeared to be largely free of altered glucuronides by HPLC, whereas the third was a complex mixture which contained altered glucuronides and III $\alpha$ and $\mathrm{XIII} \alpha$ isomers, presumably arising from intra- and intermolecular isomerization during the extensive purification procedure.

When crude BMG/BDG from rat bile was incubated $(6 \mathrm{~h}$, $37^{\circ} \mathrm{C}$ ) in aqueous HSA under argon at pH 7.0-9.0, the total pigment concentration, measured by the diazo reaction, declined by $<16 \%$ (Table III). There were, however, pronounced pH-dependent changes in pigment composition. Hydrolysis and altered glucuronide formation occurred at all pH levels but increased with increasing pH (Fig. $1, h-m$ and Table III). In addition, biliprotein was detected in all samples, with the proportion increasing markedly with increasing pH. Repetition of this experiment (at pH 7 and 9 only) with a different batch of BMG/BDG gave similar results. When BMG/BDG was incubated in phosphate $(0.1 \mathrm{M})$ buffered HSA at pH 7.4 for $28 \mathrm{~h}$ there was marked hydrolysis and rearrangement of the glucuronides without significant loss of total pigment (Table IV). The proportion of biliprotein increased with incubation time, but the overall yield was low, presumably because of the faster competing hydrolysis of starting material to unconjugated bilirubin. Similarly, incubation of BMG/BDG and purified BDG in rat serum led to rearrangement and biliprotein formation, in addition to extensive hydrolysis (Table IV). The small amount of biliprotein detected in these samples at $t$ $=0$, though close to the limits of sensitivity of the method, appeared to be real based on comparison with blank serum controls. 
Table III. Effect of pH on Formation of Biliprotein In Vitro

\begin{tabular}{|c|c|c|c|c|c|c|}
\hline \multirow[b]{2}{*}{ pH } & \multicolumn{2}{|c|}{ Total bilirubin } & \multicolumn{4}{|c|}{ \% Composition } \\
\hline & HPLC* & Diazo & $\delta$ & $\boldsymbol{\gamma}$ & $\beta$ & $\alpha$ \\
\hline & $\mathrm{mg} / 100 \mathrm{ml}$ & $\mathrm{mg} / 100 \mathrm{ml}$ & & & & \\
\hline Control & 14.2 & 17.4 & 2 & $27(34)$ & $66(66)$ & $5(1)$ \\
\hline $6.8-7.0$ & 13.7 & 12.9 & 3 & $23(36)$ & $64(57)$ & $9(7)$ \\
\hline $7.2-7.5$ & 14.0 & 14.0 & 5 & $18(37)$ & $63(50)$ & $13(13)$ \\
\hline $7.7-8.1$ & 14.9 & 15.8 & 7 & $16(22)$ & $60(62)$ & $18(15)$ \\
\hline $8.0-8.5$ & 14.6 & 15.2 & 9 & $14(21)$ & $53(55)$ & $24(25)$ \\
\hline $8.4-9.0$ & 12.1 & 15.4 & 14 & $7(19)$ & $38(41)$ & $41(40)$ \\
\hline
\end{tabular}

BMG/BDG isolated from rat bile was dissolved in unbuffered $0.5 \mathrm{mM}$ HSA (pH 7). A control sample was removed at once for analysis and the remaining solution was divided into five portions. These were titrated to $\mathrm{pH}$ values between 6.8 and 8.4 and then incubated under argon at $37^{\circ} \mathrm{C}$ in the dark for $6 \mathrm{~h}$. $\mathrm{pH}$ values given in the Table are initial and final values for each sample. * By HPLC, system B (12). $\delta$, biliprotein; $\gamma$, bilirubin diglucuronic acid esters; $\beta$, bilirubin monoglucuronic acid esters; $\alpha$, bilirubin. Values in parentheses are estimated relative proportions based on HPLC with system A; these are not corrected for biliprotein.

Markedly different results were obtained when similar experiments were carried out with conjugated bilirubin isolated by the method of Wu et al. (19). Incubation of this material $(15 \mathrm{mg} / 100 \mathrm{ml})$ in aqueous HSA (pH 7.4) resulted in hydrolysis and unconjugated bilirubin formation as observed with the BMG/BDG and BDG preparations. However, in contrast to the results obtained with the BMG/BDG preparations, substantial amounts of biliprotein were detected by HPLC in samples taken at the start of incubation, and the proportion did not increase significantly with time (e.g., $26 \%$ biliprotein at $\mathrm{t}=0 ; 27,28,31$, and $29 \%$ at $\mathrm{t}=1,3,5$ and $20 \mathrm{~h}$, respectively). Analogous results were obtained when the same material was incubated in rat serum with and without added saccharolactone $(33 \mathrm{mg} / 100 \mathrm{ml})$, except that the amounts of biliprotein detected were somewhat lower $(10-11 \%$ at $\mathrm{t}=0$; $17-19 \%$ at $t=20 \mathrm{~h})$. Similar proportions of biliprotein $(\sim 10 \%)$ were formed when other batches of bile pigment isolated from human bile by the same procedure were incubated with HSA (6-12 h). These findings indicate that conjugated bilirubin, isolated as in reference 19, which by HPLC on system $A$ contains not only BMG and BDG but also rearrangement products of these, is more reactive toward albumin than BMG/ BDG alone.

\section{Discussion}

In 1916, Hijmanns van den Bergh and Muller showed that blood from patients with obstructive jaundice contains two types of bile pigment (20). One type (direct bilirubin) reacts rapidly with diazotized sulfanilic acid; the other (indirect bilirubin) reacts more slowly. Indirect bilirubin was readily separable from plasma proteins by treatment with alcohol (8). In contrast, only part of the direct-reacting pigment was dissociable, the remainder being tightly and irreversibly attached to protein. Subsequent studies showed that the dissociable indirect-reacting pigment is unconjugated bilirubin and that the dissociable direct-reacting fraction consists principally of covalent bilirubin-glucuronic acid conjugates $(21,22)$. Conjugation of bilirubin occurs principally in the liver and the

Table IV. Formation of Biliprotein and Hydrolysis of Bilirubin Glucuronides In Vitro at $37^{\circ} \mathrm{C}$

\begin{tabular}{|c|c|c|c|c|c|c|c|}
\hline \multirow[b]{2}{*}{ Mixture } & \multirow[b]{2}{*}{ Time } & \multicolumn{2}{|c|}{ Total bilirubin } & \multicolumn{4}{|c|}{ \% Composition * } \\
\hline & & HPLC* & Diazo & $\delta$ & $\gamma$ & $\beta$ & $\alpha$ \\
\hline & $h$ & $m g / 100 \mathrm{ml}$ & $m g / 100 \mathrm{ml}$ & & & & \\
\hline \multirow{3}{*}{$\left.\begin{array}{l}\text { BDG/BMG, } \\
0.5 \mathrm{mM} \text { HSA, } \\
\text { pH } 7.4\end{array}\right\}$} & 0 & 4.5 & 4.9 & $1 \neq$ & $34(36)$ & $59(64)$ & $6(<1)$ \\
\hline & 5 & 4.2 & 4.6 & 4 & $21(22)$ & $59(59)$ & $16(19)$ \\
\hline & 28 & 4.2 & 5.1 & 6 & $5(3)$ & $33(36)$ & $56(61)$ \\
\hline \multirow{3}{*}{$\left.\begin{array}{l}\text { BDG/BMG, } \\
0.5 \mathrm{mM} \mathrm{HSA}, \\
\mathrm{pH} 7.4\end{array}\right\}$} & 0 & 4.4 & 4.5 & 1 & $30(30)$ & $62(70)$ & $7(<1)$ \\
\hline & 5 & 4.1 & 4.3 & 5 & $18(16)$ & $64(68)$ & $14(16)$ \\
\hline & 28 & 3.8 & 4.5 & 9 & $5(<1)$ & $44(44)$ & $43(56)$ \\
\hline \multirow{2}{*}{$\begin{array}{l}\text { BDG/BMG, } \\
\text { rat serum }\end{array}$} & 0 & 7.6 & 8.1 & 6 & 34 & 48 & 13 \\
\hline & 21 & 5.6 & 6.2 & 21 & 16 & 36 & 28 \\
\hline \multirow{2}{*}{$\begin{array}{l}\text { BDG, } \\
\text { rat serum } \S\end{array}$} & 0 & 2.7 & 2.8 & 9 & 45 & 36 & $10^{\prime \prime}$ \\
\hline & 4 & 2.9 & 2.7 & 21 & 27 & 35 & 18 \\
\hline
\end{tabular}

Pigments were dissolved in the designated solvent. Samples were removed after mixing $(t=0)$ and then after the stated times. * By HPLC, system B (12). $\delta$, biliprotein; $\gamma$, bilirubin diglucuronic acid esters; $\beta$, bilirubin monoglucuronic acid esters; $\alpha$, bilirubin. Values in parentheses are estimated relative proportions based on HPLC with system A; these are not corrected for biliprotein. ‡ Close to limits of detection. \$ Incubated in stoppered tubes in air; final pH 8.3-8.6. "Analysis of a sample of this solution immediately after preparation and without freezedrying and reconstitution by HPLC system A gave $100 \%$ diglucuronide and <1\% BMG or bilirubin. 
products are $\beta$-D-1-O-acyl-mono- and diglucuronides, which under normal conditions are excreted unchanged in bile (4). However, acyl glucuronides undergo base-catalyzed rearrangement to constitutional isomers at physiological pH (3-7). Such isomerization occurs with bilirubin glucuronides in vitro, even in the presence of albumin (4), and under pathological conditions in vivo $(4,5)$. Our studies provide further direct evidence that the bile pigments accumulating in the circulation during cholestasis are highly heterogeneous and they show that nonaqueous reversed-phase HPLC provides a rapid, simple method for detecting bilirubin ester heterogeneity. The heterogeneous pigments detected in serum during cholestasis were chromatographically identical to those formed on incubating BMG and BDG in vitro at physiologic pH in the presence of albumin. Because of the probable presence of large numbers of isomers ${ }^{3}$ we did not attempt to isolate or characterize these altered glucuronides which were detected by HPLC. However, their tentative identification as isomers of bilirubin glucuronides formed by acyl migration is based on the following strong circumstantial evidence: (a) They were formed, with little loss in overall pigment concentration, when authentic bilirubin glucuronides were incubated anaerobically in the dark under conditions known to induce acyl migration in bilirubin glucuronides $(3,4)$ and many other structurally analogous substances $(6,7,23)$. (b) Their formation was diminished under conditions known to inhibit glucuronide isomerization $(3,4)$. (c) They are yellow and diazo positive. (d) They migrated with authentic BMG and BDG on HPLC (system B). Furthermore, their formation in vivo or in the presence of HSA in vitro did not involve intermolecular disproportionation of bilirubin glucuronides, since the unconjugated bilirubin produced by hydrolysis or the methyl esters produced by alkaline methanolysis did not contain significant amounts of III $\alpha$ and XIII $\alpha$ isomers (A. F. McDonagh, unpublished observations). Formation of the putative glucuronide isomers in vivo during cholestasis was slow. BMG and BDG appeared first, but by 12 $h$ altered glucuronides tended to predominate. However, a stable pigment pattern with the altered glucuronides clearly predominant was not established until about 48-72 h. By then biosynthesis, acyl migration, hydrolysis, and renal excretion of the various bilirubin esters had presumably reached a steady state.

The direct-reacting bile pigment that remains with the protein precipitate upon the addition of alcohol to icteric sera was called adsorbed bilirubin by Hijmanns van den Bergh (8). Yet simple physical adsorption did not appear to be responsible for the pigment-protein association, since rigorous attempts to separate the two failed. Adsorbed bilirubin was found only in body fluids containing conjugated (direct) bilirubin. Its proportion in serum tended to be low during the early phase of obstructive jaundice but increased as jaundice receded and bilirubinemia and bilirubinuria diminished $(21,24,25)$. Later, evidence for the occurrence of an undissociable bilirubinprotein complex in pathologic sera was obtained by reversedphase chromatography (9-13), and recent studies suggest strongly that the complex contains a bilirubinoid species bonded covalently to albumin (11). Our work demonstrates that a similar biliprotein is formed during experimental cholestasis in the rat (26) and guinea pig. Within $24 \mathrm{~h}$ of biliary obstruction a substantial proportion of the bile pigment in the circulation is biliprotein and the proportion increases when the obstruction is removed. Therefore clearance of biliprotein is slower than that of bilirubin and its glucuronic acid esters, which is consistent with clinical observations $(13,27)$.

The absence of biliprotein from ligated and unligated Gunn rats and the formation of a similar substance in vitro from bilirubin glucuronides and HSA imply that biliprotein is formed in vivo by nonenzymic reactions of BMG and BDG (or derived rearrangement products) with albumin. Photochemical reactions (13) can be ruled out since our studies were conducted in the dark or under safelights. The concomitant formation of biliprotein and altered glucuronides in vitro and in vivo suggests furthermore that these two reactions compete or are interrelated. Isomerization of acyl glucuronides is an intramolecular reaction that procedes via ortho-acid intermediates (23). Similar acyl shifts from $\mathrm{O} \rightarrow \mathrm{N}$ occur readily in $O$-acylated amino-sugars (23). Albumin contains reactive $-\mathrm{NH}_{2}$ groups (28) and it is well known that aliphatic acyl esters of phenols (e.g., aspirin) can acetylate albumin by acyl transfer (29). We suggest that covalent binding of bilirubin to albumin occurs via intermolecular $\mathrm{O} \rightarrow \mathrm{N}$ migration of a bilirubin acyl group from glucuronic acid to a free $-\mathrm{NH}_{2}$ group on the protein. If this mechanism is correct, the chromophore linkage in mammalian biliprotein is a propionamide and different from that in plant and algal biliproteins (30). An alternative, but chemically less likely, mechanistic possibility is that nucleophilic attack by the -SH side-chain of albumin on a bilirubin glucuronide (or isomer) leads to acyl displacement and formation of a thioester bond (31).

Covalent interaction of $O$-acyl glucuronides with proteins has not been reported previously. We suspect that the reaction may be general and that other $O$-acyl glucuronides that bind reversibly to albumin may transfer their aglycone irreversibly to the protein if they accumulate in the circulation. Indeed, preliminary studies (Philip C. Smith, personal communication) indicate that the anti-inflammatory drug Zomepirac (6) also becomes covalently bound to albumin when its glucuronide is incubated with the protein in vitro. It is not yet known whether mammalian biliproteins have any immunological or physiological significance or whether covalent binding of bilirubin glucuronides to biomolecules other than albumin occurs in vivo. It is interesting, however, that the reaction of clofibrate $1-O$-acyl glucuronide with biomolecules is thought to contribute to the toxicity of clofibrate (31).

Rearrangement of bilirubin glucuronides in aqueous HSA was accompanied by nonenzymic hydrolysis. Contrary to a recent claim (32), hydrolysis also occurred in serum. Hydrolysis was slow at $\mathrm{pH} 7.4,37^{\circ} \mathrm{C}$, but increased markedly with $\mathrm{pH}$. Hydrolysis of aliphatic acyl esters under such mild conditions is surprising. Hydrolysis of the 1-O-acyl glucuronides might be facilitated by the adjacent pyranose oxygen, but this effect would be absent in the corresponding 2-, 3-, and 4-O-acyl 
isomers. Possibly, hydrolysis of the altered glucuronides occurs via rearrangement back to the less stable 1-O-acyl isomer. However, steric factors, neighboring - $\mathrm{OH}$ group participation, or binding to albumin might also facilitate the reaction. Whatever the mechanism, our observations suggest that spontaneous hydrolysis of bilirubin conjugates may account, at least partly, for the unconjugated bilirubin in serum of rats (Fig. 1) and patients (33) with cholestatic jaundice and for the apparent deconjugation of bilirubin glucuronides detected in experimental studies $(32,34,35)$.

Exact agreement between HPLC systems A and B would not be expected since both rely on uncorrected relative integrated peak areas and since system A ignores biliprotein. Nevertheless, comparison of analytical data from the two systems for the same samples indicated that variable, but usually slight, hydrolysis of diesters to monoesters and of monoesters to bilirubin occurred during reconstitution and pretreatment of the samples for system B (see examples in Tables III and IV). This is not surprising since pretreatment involved warming samples at $37^{\circ} \mathrm{C}, \mathrm{pH} 7.7$, for $\sim 20 \mathrm{~min}$ to remove globulins (12). Less expected was the detection of small amounts of biliprotein in solutions of BMG/BDG in serum or aqueous HSA that were lyophilized for analysis immediately after preparation and before incubation. The reason for this is unclear. One possibility is that this small amount of biliprotein was formed during lyophilization or subsequent sample manipulation. Another possibility is that the original glucuronide preparations contained small quantities of an impurity, perhaps a rearranged glucuronide species, that reacts very rapidly with protein. This is supported by the otherwise puzzling results obtained with bile pigment isolated from human bile. This material, which was shown by HPLC to have already undergone extensive isomerization during the isolation procedure, gave a substantial amount of biliprotein immediately on mixing with HSA or serum. Since biliprotein can be formed nonenzymically in vitro it is possible that the biliprotein isolated by Etter-Kjelsaas and Kuenzle (36) from human bile was formed after the excretion of bile from the liver as suggested recently by Compernolle (37). It also raises the question whether the biliprotein detected in pathologic human serum is wholly artifactual. Previous studies $(8,11$, 27) and data in this paper suggest that this is not so. However, the possibility that a small proportion of the biliprotein detected in earlier studies, particularly in samples containing high levels of bilirubin glucuronic acid esters, is artifactual cannot yet be ruled out. Certainly, careful handling of icteric sera is necessary if artifacts and changes in bile pigment composition are to be avoided.

Since the bilirubin mono- and diglucuronic acid esters in serum may be complex mixtures of isomers, HPLC methods that consolidate them into single bands may not reflect the complexity of the situation. Such methods include system B and the recently developed alkaline methanolysis procedure (33). Clearly, the proportion of mono- and diconjugates measured by these methods does not necessarily reflect the actual proportion of 1-O-acyl glucuronides (BMG and BDG) in the sample. Nor does it reflect the proportion of BMG to BDG biosynthesized in the liver, since these can subsequently undergo rearrangement, hydrolysis, covalent binding and differential renal excretion if they accumulate. Therefore, on these grounds alone, it is understandable why measurements of monoconjugate-to-diconjugate ratios by such methods have not proved diagnostically useful $(38,39)$.

HPLC system B, as used in this study, permits measurement of the biliprotein fraction in serum but consolidates the isomeric bilirubin mono- and diglucuronic acid esters into single, sometimes broadened, peaks. The nonaqueous reversedphase system A provides a somewhat clearer picture of the dissociable noncovalently bound pigment fraction but does not measure biliprotein. Of the chromatographic methods currently available for distinguishing unconjugated from conjugated hyperbilirubinemia, system $A$ is the most rapid. It is not yet known whether measuring the relative proportions of isomeric bilirubin esters in serum would be diagnostically or clinically more useful than measuring the proportion of biliprotein (13).

Note added in proof. For closely related work see Gautam, A., H. Seligson, E. R. Gordon, D. Seligson, and J. L. Boyer. 1984. Irreversible binding of conjugated bilirubin to albumin in cholestatic rats. J. Clin. Invest. 73:873-877.

\section{Acknowledgments}

We thank Dr. E. Gordon for the gift of purified bilirubin glucuronide, Ms. Diana Fedorchak and Mr. Michael Karasik for assistance in preparing the typescript, Ms. Carolyn Little for technical assistance, and Mr. Philip Smith for useful discussions and comments on the manuscript.

This work was supported in part by grants AM-26307, AM-26373, and AM-11275 from the National Institutes of Health.

\section{References}

1. Chowdhury, J. R., A. W. Wolkoff, and I. M. Arias. 1982. Heme and bile pigment metabolism. In The Liver. I. M. Arias, H. Popper, D. Schachter, and D. A. Shafritz, editors. Raven Press, New York. 309-332.

2. Haines, A. H. 1976. Relative reactivities of carboxyl groups in carbohydrates. Adv. Carbohydr. Chem. Biochem. 33:11-109.

3. Compernolle, F., G. P. Van Hees, N. B. Blanckaert, and K. P. M. Heirwegh. 1978. Glucuronic acid conjugates of bilirubin-IX $\alpha$ in normal bile compared with post-obstructive bile. Biochem. J. 185201.

4. Blanckaert, N., F. Compernolle, P. Leroy, R. van Houtte, R. Fevery, and K. P. M. Heirwegh. 1978. The fate of bilirubin-IX $\alpha$ glucuronide in cholestasis and during storage in vitro. Biochem. $J$. 171:203-204.

5. Jansen, P. L. M. 1981. $\beta$-Glucuronidase-resistant bilirubin glucuronide isomers in cholestatic liver disease-determination of bilirubin metabolites in serum by means of high-pressure liquid chromatography. Clin. Chim. Acta. 110:309-317.

6. Hasegawa, J., P. C. Smith, and L. Z. Benet. 1982. Apparent intramolecular acyl migration of zomepirac glucuronide. Drug Metab. Dispos. 10:469-473. 
7. Janssen, F. W., S. K. Kirkman, C. Fenselau, M. Stogniew, B. R. Hofmann, E. M. Young, and H. W. Rudius. 1982. Metabolic formation of $\mathrm{N}$ - and $\mathrm{O}$-glucuronides of 3-(p-chlorophenyl)thiazolo[3,2a]benzimidazole-2-acetic acid. Drug Metab. Dispos. 10:599-604.

8. Hijmanns van den Bergh, A. A. 1928. Der Gallenfarbstoff im Blute. Barth, Leipzig.

9. Kuenzle, C. C., C. Maier, and J. R. Ruttner. 1966. The nature of four bilirubin fractions from serum and of three bilirubin fractions from bile. J. Lab. Clin. Med. 67:294-306.

10. Lauff, J. J., M. E. Kaspar, and R. T. Ambrose. 1981. Separation of bilirubin species in serum and bile by high performance reversedphase liquid chromatography. J. Chromatogr. 226:391-402.

11. Lauff, J. J., M. E. Kaspar, T. W. Wu, and R. T. Ambrose. 1982. Isolation and preliminary characterization of a fraction of bilirubin in serum that is firmly bound to protein. Clin. Chem. 28:629637.

12. Lauff, J. J., M. E. Kaspar, and R. T. Ambrose. 1983. Quantitative liquid-chromatographic estimation of bilirubin species in pathological serum. Clin. Chem. 29:800-805.

13. Weiss, J. S., A. Gautam, J. J. Lauff, M. W. Sundberg, P. Jatlow, J. L. Boyer, and D. Seligson. 1983. The clinical importance of a protein-bound fraction of serum bilirubin in patients with hyperbilirubinemia. N. Engl. J. Med. 309:147-150.

14. McDonagh, A. F. 1979. Bile pigments: bilatrienes and 5,15biladienes. In The Porphyrins. D. Dolphin, editor. Academic Press, New York. 6:427-428.

15. McDonagh, A. F., and L. A. Palma. 1982. Heme catabolism in fish. Bile pigments in gallbladder bile of the electric torpedo, torpedo californicus. Comp. Biochem. Physiol. 73B:501-507.

16. McDonagh, A. F., L. A. Palma, F. R. Trull, and D. A. Lightner. 1982. Phototherapy for neonatal jaundice. Configurational isomers of bilirubin. J. Am. Chem. Soc. 104:6865-6867.

17. McDonagh, A. F. 1979. Bile pigments: bilatrienes and 5,15biladienes. In The Porphyrins. D. Dolphin, editor. Academic Press, New York. 6:461.

18. Fevery, J., B. Van Damme, R. Michiels, J. De Groote, and K. P. M. Heirwegh. 1972. Bilirubin conjugates in bile of man and rat in the normal state and in liver disease. J. Clin. Invest. 51:2482-2492.

19. Wu, T.-W., N. Zumbulyadis, S. Gross, and R. S. Gohlke. 1980. Human conjugated bilirubin-isolation, biosynthesis, and direct molecular characterization. Clin. Chem. 26:1323-1335.

20. Hymanns van den Bergh, A. A., and P. Muller. 1916. Uber eine direkte und indirekte Diazoreaktion auf Bilirubin. Biochem. $Z$. 77:90-103.

21. With, T. K. 1968. Bile Pigments. Academic Press, New York. 349-351.

22. Lathe, G. H. 1972. The degradation of heme by mammals and its excretion as conjugated bilirubin. Essays Biochem. 8:107-148.

23. Hough, L., and A. C. Richardson. 1967. The monosaccharides: pentoses, hexoses, heptoses and higher sugars. In Rodd's Chemistry of Carbon Compounds. S. Coffey, editor. Elsevier Science Publishing Co. Inc., New York. 1F:382-387.
24. Deenstra, H. 1948. On serum bilirubin during the course of an icterus. Acta Med. Scand. 132:223-237.

25. Cora, D. 1952. On the fluctuations in the diazo reaction rate and in the bilirubin adsorbed on serum proteins during the course of jaundices. Acta Med. Scand. 142:297-305.

26. Sommerhalder, Von M., C. Kuenzle, J. R. Ruttner, and C. Maier. 1962. Die 4 Serumbilirubin-Fractionen beim frischen experimentellen Verschlussikterus der Ratte. Med. Exp. 7:196-198.

27. Lauff, J. J., M. E. Kaspar, and R. T. Ambrose. 1982. In XI International Congress of Clinical Chemistry. S. Kaiser, F. Gabl, M. M. Muller, and P. M. Bayer, editors. Walter de Gruyter \& Co., New York. 995-1000.

28. Green, N. M. 1963. The apparent high reactivity of some amino groups of bovine serum albumin. Biochim. Biophys. Acta. 74:542-543.

29. Hawkins, D., R. N. Pinckard, and R. S. Farr. 1968. Acetylation of human serum albumin by acetylsalicylic acid. Science (Wash. DC). 160:780-781.

30. Schoenleber, R. W., S.-L. Leung, D. J. Lundell, A. N. Glazer, and H. Rapoport. 1983. Chromopeptides from phycoerythrins. Structure and linkage of a phycoerythrobilin tryptic tripeptide derived from a $\beta$ phycoerythrin. J. Am. Chem. Soc. 105:4072-4076.

31. Stagniew, M., and C. Fenselau. 1982. Electrophilic reactions of acyl-linked glucuronides. Drug Metab. Dispos. 10:609-613.

32. Gollan, J., L. Hammaker, V. Licko, and R. Schmid. 1981. Bilirubin kinetics in intact rats and isolated perfused liver. J. Clin. Invest. 67:1003-1015.

33. Blanckaert, N., P. M. Kabra, F. A. Farina, B. E. Stafford, L. J. Marton, and R. Schmid. 1980. Measurement of bilirubin and its monoconjugates and diconjugates in human serum by alkaline methanolysis and high-performance liquid chromatography. J. Lab. Clin. Med. 96:198-212.

34. Blanckaert, N., J. Gollan, and R. Schmid. 1980. Mechanism of bilirubin diglucuronide formation in intact rats. J. Clin. Invest. 65:1332-1342.

35. Acocella, G., L. T. Tenconi, R. Armas-Merino, S. Raia, and B. H. Billing. 1968. Does deconjugation of bilirubin glucuronide occur in obstructive jaundice. Lancet. I:68-69.

36. Etter-Kjelsaas, H., and C. C. Kuenzle. 1975. A polypeptide conjugate of bilirubin from human bile. Biochim. Biophys. Acta. 400:83-94.

37. Compernolle, F. 1982. Bilirubin conjugates: isolation, structure analysis and synthesis. In Bilirubin. K. P. M. Heirwegh and S. B. Brown, editors. CRC Press Inc., Boca Raton. 1:61

38. Scharschmidt, B. F., N. Blanckaert, F. A. Farina, P. M. Kabra, B. E. Stafford, and R. Weisiger. 1982. Measurement of serum bilirubin and its mono- and diconjugates: application to patients with hepatobiliary disease. Gut. 23:643-649.

39. Kuwaki, T. 1983. Bilirubin metabolism in obstructive jaundice with special reference to changes of bilirubin monoglucuronide in serum. Kanzo. 24:417-425; (Chem. Abstr. 1983. 99:20541). 